\title{
Comparative Study about the Efficacy of Low Level Laser Therapy and Curcumin Antimicrobial Photodynamic Therapy as a Coadjuvant Treatment of Oral Mucositis in Oncologic Patients:Antimicrobial, Analgesic and Degree Alteration Effect.
}

Rita de Cássia Dias Viana Andrade

Cruzeiro do Sul University: Universidade Cruzeiro do Sul

THAIS AZEVEDO REIS ( $\nabla$ tazevedo011@gmail.com )

Federal University of Bahia: Universidade Federal da Bahia https://orcid.org/0000-0002-9929-5763

Luciano Pereira Rosa

Federal University of Bahia: Universidade Federal da Bahia

Gabriel Pinto de Oliveira Santos

Federal University of Bahia: Universidade Federal da Bahia

Francine Cristina da Silva

Federal University of Bahia: Universidade Federal da Bahia

Research Article

Keywords: photobiomodulation, photodynamic therapy, curcumin, oral mucositis, cancer.

Posted Date: August 17th, 2021

DOl: https://doi.org/10.21203/rs.3.rs-691181/v1

License: (a) This work is licensed under a Creative Commons Attribution 4.0 International License.

Read Full License 


\section{Abstract}

INTRODUCTION: As conventional treatments currently available for mucositis are not considerably effective,there is a need to implement an adjuvant protocol for the treatment of oral mucositis in patients undergoing radiotherapy and chemotherapy.

PURPOSE: To evaluate the effect of Low Level Laser Therapy and Antimicrobial Photodynamic Therapy mediated by curcumin and blue LED as an adjunct treatment of oral mucositis foroncology patients using chemotherapy and or radiotherapy.

METHODS: Clinical, randomized study, in a single location, in an oncology service of a general hospital, with a total of 30 patients (over 18 years old) with stable oral mucosa lesions in the process of Chemotherapy and or Radiotherapy. The patients were divided into 3 groups: Control group (treated with nystatin), LLLT group (treated with Low Level Laser Therapy), and the aPDT group (treated with $450 \mathrm{~nm}$ blue LED and curcumin photosensitizer).

RESULTS: The results showed, by means of intra-group comparisons, that the two experimental treatments promoted yeast reduction of the genus Candida in the last two evaluations ( 21 days and 30 days), but not in the first two evaluations ( 7 days and 14 days). The intra-group comparisons showed that the Control and aPDT group showed a significant difference in the degree of mucositis over the four evaluations performed, with the results pointing out that the mucositis worsened in the control group from the 14th day, while reduced in the aPDT group from the 21 st day of treatment.

CONCLUSION: A reduction in the degree of mucositis and pain score was observed in the LLLT and aPDT groups, with the aPDT group standing out when presenting early clinical improvement in relation to the LLLT group and the control group, thus emphasizing its effectiveness within the desired aspects. Regarding the antimicrobial effect, aPDT showed a greater reduction of yeasts of the genus Candida in the tested parameters.

\section{Introduction}

Cancer treatment is performed through surgery, radiotherapy (RT), chemotherapy (CT), bone marrow transplantation, or even by combining more than one modality [1]. Oral mucositis is a frequent side effect, resulting from chemotherapy and radiotherapy in the treatment of cancer, associated with pain and characterized by inflammation and ulceration of the oral and gastrointestinal mucosa, making it difficult to swallow solid foods, sometimes liquids, also limiting speech and chewing [2-5]. This condition exposes the individual to generalized infections by opportunistic microorganisms and still usually triggers malnutrition and dehydration with systemic weakness, culminating in the reduction or interruption of antineoplastic therapy [2-5]. Other factors such as alcoholism and smoking, associated with chemotherapy and/or radiotherapy, fungal infections and poor oral hygiene can increase or worsen the condition $[4,6,7]$. 
There are no universally defined treatment protocols for oral mucositis. Many procedures have been and are used, such as oral hygiene care, use of oral rinses, analgesics, antibiotics, cryotherapy, local anesthetics, growth factors and cytokines, anti-inflammatory agents, among others [8].

It is observed that Low Level Laser Therapy (LLLT) reduces pain, inflammation and edema, promotes healing of deeper tissues and nerves, and has good results for the treatment of oral mucositis in cancer patients, associated with the photobiomodulation process ${ }^{9}$. Another therapy widely usedis the Antimicrobial Photodynamic Therapy (aPDT) based on the activation of photosensitizing agents with a light at a specific wavelength ${ }^{1}$. The light (laser or LED) activates a photosensitizer that is applied to the target, thus, its toxic effect is explained in two ways: by the process of redox and by the formation of singlet oxygen $[4,5,9-12]$. Among the different types of photosensitive agents used in aPDT, curcumin and curcuminoids have been gaining space in the research fields. These compounds are derived from turmeric, a natural yellow pigment that consists of a mixture of three curcuminoids: curcumin, demetoxicurmine and bis-demetoxicurcumin. In addition to their use as a photosensitizing agent in aPDT, these compounds have pharmacological properties such as: anti-inflammatory, antioxidant, anticancer, antibacterial and antifungal [13].

Thus, in the present study, we proposed to compare the effectiveness of LLLT, aPDT mediated by curcumin and blue light as an adjunct to the treatment of cancer patients using chemotherapy and or radiotherapy with oral mucositis. In addition, this work also aimed to contribute to the knowledge about the clinical characteristics of patients in the studied sample, to evaluate the effectiveness of aPDT mediated by curcumin and blue light in the elimination of yeast of the genus Candida in saliva samples, and to evaluate the effectiveness ofLLLT in the clinical improvement of ulcers in patients with oral mucositis.

\section{Methods}

\section{Ethical aspects and study location}

The study was approved by the Human Research Ethics Committee of the Multidisciplinary Health Institute of the Federal University of Bahia, under number CAAE 83784618.4.0000.5556. The study was carried out at the High Complexity Assistance Unit in Oncology of the General Hospital of Vitória da Conquista (HGVC) in the city of Vitória da Conquista, Bahia, Brazil.

\section{Population}

The population chosen for this study was composed of cancer patients,submitted to chemotherapy and/or radiotherapy, who seek the Dentistry service of HGVC. The sample calculation showed the need for a sample ofthirty patients with a $95 \%$ confidence interval and a sampling error of $5 \%$.

The following inclusion criteria were used for the research: 1) be 18 years or older; 2) have stable lesions in the process of $\mathrm{CT}$ and/or RT; 3 ) Consent to participate in the research by signing the Informed Consent 
Form. The exclusion criteria were: 1) Patients who were receiving medication for the treatment and prevention of mucositis; 2) Patients unable to comply with the treatment procedure or the oral hygiene protocol.

\section{Statistical Analysis}

For the descriptive analysis of the data, the mean, standard deviation and absolute and relative frequencies of the groups were used. Data normality was tested using the ShapiroWilk test and homoscedasticity using Levene's test. Comparisons of demographic and clinical characteristics between experimental groups were made using Fisher's exact test (for sex) and one-way analysis of variance (oneway ANOVA). The immediate effect of treatments on the yeast response of the genus Candida was assessed using the Wilcoxon and t- Student tests for paired samples (for intra-group comparison) and the Mann-Whitney and t-Student tests for independent samples (for intergroup comparison). To verify the late effect of treatments on the yeast response of the genus Candida, mucositis degree and pain, nonparametric statistic testswere used: Friedman test for intra-group comparisons (with comparisons between pairs being tested by the Wilcoxon test) and Kruskal-Wallis test for intergroup comparisons (with comparisons between pairs being tested by the Mann-Whitney test). The level of significance adopted in all analyzes was $5 \%(a=0.05)$. The data were tabulated and analyzed using IBM SPSS Statistics for Windows (IBM SPSS. 21.0, 2012, Armonk, NY: IBM Corp.).

\section{Patient Follow-up Care, Safety, and Outcome Measures}

The patients included in the study had the characteristics collected: sex, age, type of cancer, location of initial cancer, type of treatment, number of lesions, location of the mucositis according to the anatomical region of the oral mucosa, and respective degree of mucositis(according to World Health Organization WHO: 1- burning, erythema;2- erythema, ulcer, solid diet; - 3- confluence of ulcers, liquid diet; 4- oral feeding is not possible, liquid diet). The data were recorded on individual records.

Samples of unstimulated saliva were collected before and after the proposed treatments. The collection was performed with sterile universal collectors for 1 minute and then identified in coded form. Inside the laminar flow, in an aseptic environment, the saliva samples were processed through the decimal dilution of $10^{-1}, 10^{-2}$ and $10^{-3} .100 \mu \mathrm{L}$ aliquots of the initial samples and dilutions obtained were seeded in duplicate in Petri dishes containing Sabouraud dextrose agar culture medium plus $0.1 \mathrm{mg} / \mathrm{mL}$ of chloramphenicol for the growth of yeast species of the genus Candida spp. The plates were incubated at $37^{\circ} \mathrm{C}$ for 48 hours in an Oxygen Chemical Demand (BOD) oven. When there was no growth during this period, the samples were left for another five days at room temperature. After growth, the colonies were examined for morphological characteristics (size, shape, surface, halos and presence of pigments), and the number of colonies forming units per milliliter $(\mathrm{CFU} / \mathrm{mL})$ was counted.

The evaluation of the analgesic effect was performed through the application of the Visual Analogic Scale (VAS) for pain. This assessment was carried out immediately before the patients started the proposed treatments and was always repeated after the completion of each procedure throughout the study period. 
The assessment of clinical improvement of mucositis lesions was performed after each procedure once a week taking into account a graded classification proposed by the WHO.

\section{Treatment Protocol}

Patients $(n=30)$ were divided into three groups $(n=10)$ : LLLT, aPDT and Control (standard treatment protocol with nystatin).

For the aPDT group, the photosensitizing agent was prepared at the time of use with the amount of 7.5 $\mathrm{mg}$ of curcumin (Sigma-Aldrich, St. Louis, USA). The curcumin solution $(10 \mathrm{ml})$ that was sprayed inside the oral cavity remained for 10 minutes for impregnation or pre-irradiation time, with the mouth closed to prevent the incidence of ambient light. After the photosensitizing agent was impregnated, the oral cavity was illuminated with a blue diode light emitter (Prototype, Project Finep/Gnatus LED Edixeon, Edison Opto Corporation, New Taipei City, Taiwan), with an intensity of $67 \mathrm{~mW} / \mathrm{cm}^{2}$, central focus showing a wavelength of $450 \mathrm{~nm}$ and an estimated average fluency of $20.1 \mathrm{~J} / \mathrm{cm}^{2}$ for 10 minutes of application.

LLLT was performed with a Therapy EC equipment (DMC, São Carlos, São Paulo, Brazil), wavelength of $660 \mathrm{~nm}$ red in a punctual and continuous manner, beam diameter of $0.25 \mathrm{~cm}^{2}$, power of $100 \mathrm{~mW}$, irradiation time of 03 seconds per point, generating a fluency of $1.2 \mathrm{~J} / \mathrm{cm}^{2}$ over the entire length of the lesion with an equidistance of $1 \mathrm{~cm}$ between the points. The light was applied with the tip of the equipment in contact with the lesions. LLLT was performed 3 times a week for 30 days or the period in which the patient was undergoing radiation and/or chemotherapy.

All patients received the proposed treatments for a period of 30 days. After this period, there was a followup for another 30 days to check for possible recurrence of the lesions or worsening of the condition.

\section{Results}

In the first stage of this study, patients had information collected about their health conditions and sociodemographic variables. The analysis of demographic characteristics did not show any significant difference between the groups regarding the distribution between sexes and mean age (Table 1). 
Table 1

Demographic and clinical characteristics of the sample in the baseline, according to the experimental groups.

\begin{tabular}{|c|c|c|c|c|}
\hline & Control & LLLT & aPDT & ${ }^{*}$ p-value \\
\hline \multicolumn{5}{|l|}{ Sex } \\
\hline Male & $10(100,0 \%)$ & $8(80,0 \%)$ & $8(80,0 \%)$ & \multirow[t]{2}{*}{0,507} \\
\hline Female & $0(0,0 \%)$ & $2(20,0 \%)$ & $3(20,0 \%)$ & \\
\hline \multicolumn{5}{|c|}{ Age of population } \\
\hline Age (years) & $60,1 \pm 10,6$ & $57,3 \pm 7,2$ & $60,5 \pm 6,8$ & 0,655 \\
\hline
\end{tabular}

The immediate effect of LLLT and aPDT on theCandida yeast response over different treatment sessions can be seen in Table 2. It was found through intra-group comparisons that the two treatments reduced Candida yeast in both treatments last evaluations ( 21 days and 30 days), but not in the first two ( 7 days and 14 days). However, intergroup comparisons indicated that the average percentage of yeast reduction in the genus Candida after the application of therapies was significantly higher in the aPDT group in all four assessments $(7,14,21$ and 30 days). The late effect of treatments on the yeast response of the genus Candida, degree of mucositis and pain are shown in Table 2. It was observed through intra-group comparisons that none of the groups showed a statistical difference in response in the yeast of the genus Candida between the four evaluations performed. However, intergroup comparisons indicated a significant difference in the third ( 21 days) and fourth (30 days) evaluations, with the results pointing out that, compared to the control group, the LLLT group had a lower number of colony-forming units in the last evaluation (30 days) and the aPDT group had the lowest number in the last two evaluations (21 and 30 days). There were no statistical differences between the LLLT and aPDT groups in the bacterial response. 
Table 2

Acute effect of low-level laser therapy and curcumin-mediated antimicrobial photodynamic therapy on the yeast response (genus Candida), over different treatment sessions.

\section{LLLT aPDT}

7 days

Afeter therapy

$398,90 \pm 416,03$

$(\mathrm{UFC} / \mathrm{mL})$

Before therapy

$384,90 \pm 416,52$

(UFC/mL)

$\Delta \%$

$-4,0 \pm 14,0$

$-39,3 \pm 35,3 \uparrow$

14 days

Afeter therapy

$410,10 \pm 462,54$

$370,10 \pm 398,67$

(UFC/mL)

Before therapy

$389,90 \pm 448,99$

(UFC/mL)

$\Delta \%$

$-2,5 \pm 21,5$

$-35,3 \pm 67,5^{\star *}$

21 days

Afeter therapy

$363,60 \pm 395,09$

$294,10 \pm 251,53$

(UFC/mL)

Before therapy

$343,20 \pm 381,68$ *

$154,40 \pm 172,47$ *

(UFC/mL)

$\Delta \%$

$-9,4 \pm 7,4$

$65,6 \pm 26,2 \uparrow$

30 days

Afeter therapy

$268,40 \pm 290,20$

$342,30 \pm 647,07$

(UFC/mL)

Before therapy

$250,30 \pm 275,87 \uparrow$

$218,80 \pm 472,24$ *

(UFC/mL)

$\Delta \%$

$-4,3 \pm 10,1$

$-51,9 \pm 24,4 \uparrow$ 


\section{LLLT}

aPDT

UFC, Colony Forming Units; $\Delta \%$ percentage difference; LLLT, Low Intensity Laser Therapy; aPDT, Antimicrobial Photodynamic Therapy. * Intragroup difference $(p<0.05$, Wilcoxon test); $\uparrow$ Intragroup difference $(p<0.05$, t test for paired samples); 1 Intergroup difference $(p<0.05, t$ test for independent samples); $* \star$ Intergroup difference $(p<0.05$, Mann-Whitney test). Values are expressed as mean \pm standard deviation.

The intra-group comparisons showed that the Control and aPDT groups showed a significant difference in the degree of mucositis over the four evaluations performed, with the results pointing out that mucositis worsened in the control group from the 14th day, while it decreased in the aPDT group from the 21 st day of treatment. Intergroup comparisons indicated statistical difference in the third (21 days) and fourth (30 days) evaluations, with the results showing that, compared to the control group, the LLLT group had a lower degree of mucositis in the last evaluation (30 days) and the aPDT group in the last two evaluations ( 21 and 30 days). There were no statistical differences between the LLLT and aPDT groups in the degree of mucositis (Table 3). The results of the intra-group comparisons indicated that all three groups showed a statistical difference in the pain score over the four evaluations performed, with the results demonstrating that in the LLLT group there was pain reduction after 21 days of treatment, while in the aPDT group this effect was observed earlier (14 days); in the control group there was a reduction in pain in the third assessment ( 21 days), followed by an increase in the last assessment ( 30 days). Intergroup comparisons showed a greater analgesic effect of treatments with LLLT and aPDT at the end of the study (30 days), compared to the control group (Table 3 ). 
Table 3

Chronic effects of treatments on Candida yeast response, degree of mucositis and pain.

\begin{tabular}{|c|c|c|c|c|}
\hline & Control & LLLT & aPDT & $\uparrow p-v a l u e$ \\
\hline \multicolumn{5}{|c|}{ Candida (UFC/mL) } \\
\hline 7 Days & $463,60 \pm 316,12$ & $384,90 \pm 416,52$ & $255,3 \pm 206,86$ & 0,358 \\
\hline 14 Days & $535,70 \pm 447,85$ & $389,90 \pm 448,99$ & $311,50 \pm 349,69$ & 0,338 \\
\hline 21 Days & $480,70 \pm 276,75$ & $343,20 \pm 381,68$ & $154,4 \pm 172,47 \uparrow$ & 0,013 \\
\hline 30 Days & $536,50 \pm 342,49$ & $250,30 \pm 275,87 \uparrow$ & $218,80 \pm 472,24 \uparrow$ & 0,008 \\
\hline *p-value & 0,218 & 0,566 & 0,781 & \\
\hline \multicolumn{5}{|c|}{ Degree of mucositis } \\
\hline 7 Days & $1,90 \pm 0,88^{a}$ & $1,50 \pm 0,53$ & $2,30 \pm 1,16^{a b}$ & 0,230 \\
\hline 14 Days & $2,70 \pm 0,95^{b}$ & $1,70 \pm 1,06$ & $2,30 \pm 1,06^{\mathrm{a}}$ & 0,149 \\
\hline 21 Days & $2,90 \pm 0,74^{b}$ & $1,90 \pm 1,10$ & $1,70 \pm 0,68^{\mathrm{b}} \mathfrak{}$ & 0,013 \\
\hline 30 Days & $2,90 \pm 0,88^{b}$ & $1,50 \pm 0,85 \uparrow$ & $1,20 \pm 0,42^{\mathrm{C}} \uparrow$ & $<0,001$ \\
\hline *p-value & 0,003 & 0,166 & 0,003 & \\
\hline \multicolumn{5}{|c|}{ Pain (EVA) } \\
\hline 7 Days & $7,80 \pm 0,79^{a}$ & $6,80 \pm 1,03^{a}$ & $8,10 \pm 0,99^{a \star \star}$ & 0,029 \\
\hline 14 Days & $7,50 \pm 0,85 a$ & $6,20 \pm 1,40 a b$ & $6,30 \pm 1,57 b$ & 0,073 \\
\hline 21 Days & $6,50 \pm 0,71^{b}$ & $5,50 \pm 1,35^{b c}$ & $4,90 \pm 1,91^{c}$ & 0,096 \\
\hline 30 Days & $7,40 \pm 0,70^{a}$ & $4,90 \pm 1,20^{\mathrm{C}} \uparrow$ & $4,00 \pm 1,89^{\mathrm{C}} \uparrow$ & $<0,001$ \\
\hline${ }^{\star} p$-value & 0,003 & 0,001 & $<0,001$ & \\
\hline \multicolumn{5}{|c|}{$\begin{array}{l}\text { UFC, Colony Forming Units; LLLT, Low Level Laser Therapy; aPDT, Antimicrobial Photodynamic } \\
\text { Therapy; EVA, Visual Analogue Scale. * Friedman test: a, } b \text {, c different superscript letters horizontally } \\
\text { indicate significant intra-group difference between treatment days. }(p<0.05 \text {, Wilcoxon test); } \uparrow \text { Kruskal- } \\
\text { Wallis test: significant difference in relation to the control group and } \star \star \text { significant difference in } \\
\text { relation to the LLLT groups ( } p<0.05 \text {, Mann-Whitney test). Values are expressed as mean } \pm \text { standard } \\
\text { deviation }\end{array}$} \\
\hline
\end{tabular}

\section{Discussion}

Cancer is a major cause of morbidity and mortality worldwide, with incidence rates that vary widely depending on geographic location, age, sex and race. Low- and middle-income countries have a 
proportionally higher burden of cancer than high-income countries, but their health systems are not prepared to deal with this problem [14].

For Brazil, it is estimated that 11.200 new cases of oral cavity cancer in men and 3.500 in women are estimated for each year of the 2018-2019 biennium. These numbers correspond to an estimated risk of 10.86 new cases per 100.000 men, ranking fifth; and 3.28 for every 100.000 women, being the 12 th most common among all cancers ${ }^{14}$. Of these patients, it is common to develop oral side effects as a result of the treatment to which they are submitted, since changes in the oral microbiota, changes in physiological functions and/or important structural changes are inherent to the treatment and end up causing side effects such as: xerostomia, osteoradionecrosis, candidiasis, mucositis, among other several found in the literature [15-17].

The most important side effects of antineoplastic therapy in the past are vomiting and decreased immunity due to myelosuppression, however, due to the use of antibiotics and the use of hematopoietic agents, vomiting and immune compromise have been greatly reduced [15-17]. However, mucositis has recently emerged as one of the most serious side effects in cancer treatment $[4,5]$. When oral mucositis develops during cancer treatment, it can lead to dysphagia, pain, changes in taste, vomiting, nausea, decreased food intake, fatigue and weight loss, in addition, discontinuation of therapy due to mucositis can lead to an extension of the period of the same, which can affect the result obtained or in the deterioration of the quality of life $[4,5]$. The purpose of rehabilitation is to restore the patient to normal life, promoting the recovery of physical, emotional, mental and social function[18].

When analyzing the results achieved, it is observed that the two treatments reduced Candida yeast in the last two evaluations ( 21 days and 30 days), but not in the first two (7 days and 14 days). However, intergroup comparisons indicated that the average percentage of yeast reduction of the genus Candida after the application of therapies was significantly higher in the aPDT group in all four assessments ( 7 , 14,21 and 30 days).

Regarding the antimicrobial efficacy of aPDT, many studies have shown promising results, often reaching inactivation rates of more than $5 \log 10 \mathrm{CFU}$, which is understood as a disinfectant effect according to infection control guidelines. Data suggest that photodynamic therapy is potentially effective against bacterial, viral, fungal and protozoal infections [19-22]. The in vitro mechanism of action described in some studies shows that this therapy induces the generation of reactive oxygen (ROS) and nitrogen species, which effectively damage a variety of fungal cell structures and induce cell death [23-25].

The intra-group comparisons demonstrated that the control and aPDT groups showed a significant difference in the degree of mucositis over the four evaluations carried out, with the results pointing out that the mucositis worsened in the control group from the 14th day, while reduced in the aPDT group from the 21th day of treatment. Intergroup comparisons indicated statistical difference in the third (21 days) and fourth (30 days) evaluations, with the results showing that, compared to the control group, the LLLT group had a lower degree of mucositis in the last evaluation (30 days) and the aPDT group in the 
last two evaluations (21 and 30 days). No statistical differences were observed between the LLLT and aPDT groups in the degree of mucositis, demonstrating that both therapies are effective in the treatment of mucositis.

A Systematic Review and Meta-Analysis conducted in 2014 demonstrated that prophylactic LLLT reduces the overall risk of severe mucositis and other measures of mucositis severity, including the duration of severe mucositis in cancer patients and those undergoing aPDT [13]. Numerous studies carried out by scientists have shown that LLLT normalizes microcirculation, activates endothelocytes, stimulating their functional activity by dilating and opening reserve capillaries, providing oxygen access to epithelial cells and promoting activation of cell metabolism [26]. Different studies have described a decrease in the incidence and severity of mucositis, apparently due to accelerated regeneration and affected tissue healing, thereby reducing inflammation and pain [9]. The application of LLLT accelerates oral reepithelization, favorably influencing the results of oral mucositis in patients undergoing hematopoietic stem cell transplantation and myeloablative chemotherapy [7].

The results of the intra-group comparisons indicated that all three groups showed a statistical difference in the pain score over the four evaluations performed, with the results demonstrating that in the LLLT group there was pain reduction after 21 days of treatment, while in the aPDT group this effect was observed earlier (14 days); in the control group there was a reduction in pain in the third assessment (21 days), followed by an increase in the last assessment (30 days). Intergroup comparisons showed a greater analgesic effect of treatments with LLLT and aPDT at the end of the study (30 days), compared to the control group. The results presented here clearly demonstrate the effectiveness of these treatment modalities, proving to be a valid option for improving patients' quality of life.

Pain associated with mucositis often impairs functional status, this painful side effect reduces quality of life and often requires narcotic analgesia, enteral or parenteral nutrition at additional costs [27].

It is noteworthy that, to date, a large number of reliable studies, which prove the effectiveness of LLLT in the treatment of mucositis after chemotherapy, have been published, and they report a reduction in the likelihood of complications, in the severity of the disease and in the pain level. The preventive use of LLLT does not allow oral mucositis to develop, significantly reducing the cost of maintaining patients in the hospital and the total cost of treatment, the high efficiency of LLLT being explained by numerous mechanisms of laser biomodulation [26].

\section{Conclusion}

aPDT mediated by curcumin and LLLT are a non-invasive modality for the prevention and management of oral mucositis with some advantages such as antimicrobial effect, analgesic effect and reduction of inflammation.

A reduction in the degree of mucositis and pain score was observed in the LLLT and aPDT groups, with the aPDT group standing out when presenting early clinical improvement in relation to the LLLT group 
and the control group, thus emphasizing its effectiveness within the desired aspects. However, in aPDT mediated by curcumin when compared with LLLT, a greater reduction of Candida yeasts was noticed. In addition, the average fluency of $20.1 \mathrm{~J} / \mathrm{cm}^{2}$ irradiated by LED associated with curcumin was effective in lethal sensitization of yeasts of the genus Candida.

\section{Declarations}

\section{Financing}

Not applicable

\section{Availability of data and materials}

Not applicable

\section{Code availability}

Not applicable

\section{Conflict of interest}

The authors declare that they have no conflict of interest.

\section{Author contributions}

All authors contributed to the study conception and design. Material preparation, data collection and analysis were performed by Rita de Cássia Dias Viana Andrade, Thaís Azevedo Reis and Francine Cristina da Silva. The first draft of the manuscript was written by Rita de Cássia Dias Viana Andrade and all authors commented on previous versions of the manuscript. All authors read and approved the final manuscript.

\section{Ethical aspects and study location}

The study was approved by the Human Research Ethics Committee of the Multidisciplinary Health Institute of the Federal University of Bahia, under number CAAE 83784618.4.0000.5556. The study was carried out at the High Complexity Assistance Unit in Oncology of the General Hospital of Vitória da Conquista (HGVC) in the city of Vitória da Conquista, Bahia, Brazil.

\section{Consent to participate}

Not applicable

\section{Consent to publication}

Not applicable 


\section{Acknowledgment}

We would like to thank Prof. Dr. Walter João Genovese (in memoriam) for his immense contribution in the area of laser therapy. His work, especially in Dentistry, has always been and will be a source of inspiration for many researchers and clinicians.

\section{References}

1. Cheng KK, Leung SF, Liang RH, Tai JW, Yeung RM, Thompson DR (2010) Severe oral mucositis associated with cancer therapy: impact on oral functional status and quality of life. Support Care Cancer 18(11):1477-85. doi:10.1007/s00520-009-0771-7

2. Scully C, Porter S (2000) Oral cancer. The Bmj 321(7253):97-100. doi: 10.1136/bmj.321.7253.97

3. Spijkervet FK, van Saene HK, Panders AK, Vermey A, Mehta DM (1989) Scoring irradiation mucositis in head and neck cancer patients. J Oral Pathol Med 18(3):167-71. doi:10.1111/j.16000714.1989.tb00756.x

4. Köstler WJ, Hejna M, Wenzel C, Zielinski CC (2001) Oral mucositis complicating chemotherapy and/or radiotherapy: options for prevention and treatment. CA: a cancer journal for clinicians 51(5):290-315. doi.org/10.3322/canjclin.51.5.290

5. Trotti A, Bellm LA, Epstein JB, et al (2003) Mucositis incidence, severity and associated outcomes in patients with head and neck cancer receiving radiotherapy with or without chemotherapy: a systematic literature review. Radiother Oncol 66(3):253-62. doi:10.1016/s0167-8140(02)00404-8

6. Dörr W, Hamilton CS, Boyd T, Reed B, Denham JW (2002) Radiation-induced changes in cellularity and proliferation in human oral mucosa. International Journal of Radiation Oncology* Biology* Physics 52(4):911-917. doi.org/10.1016/S0360-3016(01)02721-3

7. Sonis ST, Elting LS, Keefe D, et al (2004) Perspectives on cancer therapy-induced mucosal injury: pathogenesis, measurement, epidemiology, and consequences for patients. Cancer 100 (9 Suppl):1995-2025. doi:10.1002/cncr.20162

8. Cardoso MdFA, Novikoff S, Tresso A, Segreto RA, Cervantes O (2005) Prevenção e controle das seqüelas bucais em pacientes irradiados por tumores de cabeça e pescoço. Radiologia brasileira 38(2):107-115. doi.org/10.1590/S0100-39842005000200006

9. Fekrazad R, Chiniforush N (2014) Oral mucositis prevention and management by therapeutic laser in head and neck cancers. J Lasers Med Sci. Winter 5(1):1-7.

10. Kelner N, Castro JFLd (2007) Laser de baixa intensidade no tratamento da mucosite oral induzida pela radioterapia: relato de casos clínicos. Rev Bras Cancerol. 53(1):29-33. doi.org/10.32635/21769745.RBC.2007v53n1.1825

11. Caccelli ÉMN, Pereira MdLM, Rapoport A (2009) Avaliação da mucosite e xerostomia como complicações do tratamento de radioterapia no câncer de boca e orofaringe. Rev Bras Cir Cabeça Pescoço 38(2):80-83. 
12. Fonseca MB, Junior PO, Pallota RC, et al (2008) Photodynamic therapy for root canals infected with Enterococcus faecalis. Photomed Laser Surg 26(3):209-13. doi:10.1089/pho.2007.2124

13. Suzuki IL (2016) Viabilização da curcumina natural nanoencapsulada para inativação fotodinâmica. Dissertação, Universidade de São Paulo

14. Vieira ACF, Lopes FF (2006) Mucosite oral: efeito adverso da terapia antineoplásica. Revista de Ciências Médicas e Biológicas 5(3):268-274. doi:10.9771/cmbio.v5i3.4135

15. Freitas DA, Caballero AD, Pereira MM, Oliveira SKM, Silva GPE, Hernández CIV (2011) Sequelas bucais da radioterapia de cabeça e pescoço. Revista CEFAC 13(6):1103-1108. doi.org/10.1590/S1516-18462011005000071

16. Jham BC, Freire ARdS (2006) Complicações bucais da radioterapia em cabeça e pescoço. Revista brasileira de otorrinolaringologia 72(5):704-708. doi: 10.1590/S0034-72992006000500019

17. Lôbo ALG, Martins GB (2009) Consequências da radioterapia na região de cabeça e pescoço: uma revisão da literatura. Revista Portuguesa de Estomatologia, Medicina Dentária e Cirurgia Maxilofacial 50(4):251-255. doi.org/10.1016/S1646-2890(09)70026-3

18. Karu $T$ (1988) Molecular mechanism of the therapeutic effect of low-intensity laser radiation. Lasers Life Sci 2(1):53-74.

19. Cristina Da Silva F, Rosa LP, Santos GPDO, et al (2020) Total mouth photodynamic therapy mediated by blue led and curcumin in individuals with AIDS. Expert Review of Anti-infective Therapy 1-8. doi:10.1080/14787210.2020.1756774

20. Araújo TSD, Rodrigues PLF, Santos MS, et al (2018) Reduced methicillin-resistant Staphylococcus aureus biofilm formation in bone cavities by photodynamic therapy. Photodiagnosis and photodynamic therapy. 21:219-223.doi: 10.1016/j.pdpdt.2017.12.011

21. Almeida PP, Pereira IS, Rodrigues KB, et al (2017) Photodynamic therapy controls of Staphylococcus aureus intradermal infection in mice. Lasers Med Sci 32(6):1337-1342. doi:10.1007/s10103-0172247-1

22. Tanajuraa BR, Rosa FCS, Inadab NM, Bagnato VS, Rosa LP (2018) Effectiveness of the Association of Cellulose Membrane Dressing and Photodynamic Therapy Mediated by Curcumin to Reduce Contamination and Prevent Recontamination in Pressure Lesions. Therapy 1(3):121.

23. Jerjes W, Upile T, Hamdoon Z, Mosse CA, Akram S, Hopper C (2011) Photodynamic therapy outcome for oral dysplasia. Lasers Surg Med 43(3):192-9. doi:10.1002/lsm.21036

24. Daniell MD, Hill JS (1991) A history of photodynamic therapy. N Z J Surg. 61(5):340-8. doi:10.1111/j.1445-2197.1991.tb00230.x

25. Nunez S, Ribeiro MS, Segundo A (2012) Laser de baixa potência: princípios básicos e aplicações clínicas na odontologia. Elsevier Brasil.

26. Agostinis P, Berg K, Cengel KA, et al (2011) Photodynamic therapy of cancer: an update. CA Cancer J Clin 61(4):250-81. doi:10.3322/caac.20114 
27. Castano AP, Demidova TN, Hamblin MR (2004) Mechanisms in photodynamic therapy: part onephotosensitizers, photochemistry and cellular localization. Photodiagnosis Photodyn Ther 1(4):27993. doi:10.1016/S1572-1000(05)00007-4 